\title{
Approximation of the mixed additive and cubic functional equation in paranormed spaces
}

\author{
Zhihua Wanga,*, Prasanna K. Sahoo ${ }^{\mathrm{b}}$ \\ a School of Science, Hubei University of Technology, Wuhan, Hubei 430068, P. R. China. \\ ${ }^{b}$ Department of Mathematics, University of Louisville, Louisville, KY 40292, USA.
}

Communicated by S.-M. Jung

\begin{abstract}
In this paper, we prove some theorems about the Hyers-Ulam stability of the functional equation

$$
f(2 x+y)+f(2 x-y)=2 f(x+y)+2 f(x-y)+2[f(2 x)-2 f(x)]
$$

in paranormed spaces. From these theorems, as corollaries, we obtain the stability of the above functional equation with weaker conditions controlled by product of powers of norms and mixed-type product-sum of powers of norms. (C)2017 All rights reserved.
\end{abstract}

Keywords: Additive map, cubic map, Hyers-Ulam stability, paranormed space. 2010 MSC: 39B82, 39B72, 35A17, 47H10.

\section{Introduction}

In 1940, Ulam [18] posed the first stability problem concerning group homomorphisms: let $\left(\mathrm{G}_{1}, *\right)$ be a group and $\left(\mathrm{G}_{2}, \diamond, \mathrm{d}\right)$ be a metric group with the metric $\mathrm{d}(\cdot, \cdot)$. Given $\varepsilon>0$, does there exist a $\delta(\varepsilon)>0$ such that if a mapping $f: G_{1} \rightarrow G_{2}$ satisfies the inequality

$$
d(f(x * y), f(x) \diamond f(y))<\delta
$$

for all $x, y \in G_{1}$, then there is a homomorphism $g: G_{1} \rightarrow G_{2}$ with $d(f(x), g(x))<\varepsilon$ for all $x \in G_{1}$ ?

In 1941, Hyers [4] gave the first affirmative partial answer to the question of Ulam for Banach spaces. Hyers' result was generalized by Aoki [1] for additive mappings and by Rassias [14] for linear mappings. In 1982, Rassias [15] considered a further generalization of the results of Hyers and proved a theorem using weaker conditions controlled by a product of different powers of norms. Forti [2] and Găvruţa [3] obtained generalized Rassias' result which allows the Cauchy difference to be controlled by a general unbounded function in the spirit of Rassias' approach. Since then, the stability of several functional equations has been extensively investigated by several mathematicians (see $[5-7,16,17]$ and references

\footnotetext{
*Corresponding author

Email addresses: matwzh2000@126.com (Zhihua Wang), sahoo@louisville.edu (Prasanna K. Sahoo)
} 
therein). Recently, some stability results concerning with the linear functional equations such as the additive Cauchy functional equation, quadratic functional equation, the cubic functional equation, the quartic functional equation, and the mixed type additive-quadratic-cubic-quartic functional equation (cf. $[8,10-13])$ in paranormed spaces have been obtained.

In 2008, Najati and Eskandani [9] considered the functional equation

$$
f(2 x+y)+f(2 x-y)=2 f(x+y)+2 f(x-y)+2[f(2 x)-2 f(x)]
$$

with $f(0)=0$. It is easy to show that the mapping $f(x)=a x^{3}+c x$ is a solution of the functional equation (1.1). They determined the general solution of the functional equation (1.1) and also investigated the Hyers-Ulam-Rassias stability of functional equation (1.1) in quasi-Banach spaces.

The main purpose of this paper is to investigate the generalized Hyers-Ulam stability of functional equation (1.1) in paranormed spaces. We also present stability of functional equation (1.1) using weaker conditions controlled by product of powers of norms and mixed-type product-sum of powers of norm.

\section{Definitions}

In this section, we present some definitions that will be used throughout this paper. Let us begin with the definition of paranorm.

Definition 2.1. Let $X$ be a vector space. A paranorm $P: X \rightarrow[0, \infty)$ is a function on $X$ such that

(1) $\mathrm{P}(0)=0$;

(2) $P(-x)=P(x)$;

(3) $P(x+y) \leqslant P(x)+P(y)$ (triangle inequality);

(4) If $\left\{t_{n}\right\}$ is a sequence of scalars with $t_{n} \rightarrow t$ and $\left\{x_{n}\right\} \subset X$ with $P\left(x_{n}-x\right) \rightarrow 0$, then $P\left(t_{n} x_{n}-t x\right) \rightarrow 0$ (continuity of multiplication).

The pair $(\mathrm{X}, \mathrm{P})$ is called a paranormed space and $\mathrm{P}$ is a paranorm on the vector space $\mathrm{X}$.

The paranorm is called total if, in addition, we have $P(x)=0$ implies $x=0$. A Fréchet space is a total and complete paranormed space. Throughout this paper, we assume that $(X, P)$ is a Fréchet space and $(Y,\|\cdot\|)$ is a Banach space. It is easy to see that if $P$ is a paranorm on $X$, then $P(n x) \leqslant n P(x)$ for all $x \in X$ and $n \in \mathbb{N}$.

Let $\mathrm{V}$ and $\mathrm{W}$ be vector spaces, and $\mathrm{V}=\mathrm{V} \times \mathrm{V} \times \cdots \times \mathrm{V}$ be the Cartesian product of $n$ copies of $\mathrm{V}$. A mapping $A: \mathcal{V} \rightarrow W$ is called an $n$-additive mapping if and only if $A$ is additive in each variable. The diagonal of a 3-additive mapping is called a cubic mapping whereas the diagonal of a 2-additive mapping is called a quadratic mapping.

For a given mapping $f: X \rightarrow Y$, where $X$ is either a paranorm or a norm space and $Y$ is a norm or a paranorm space, we define $D_{f}: X \times X \rightarrow Y$ by

$$
D_{f}(x, y)=f(2 x+y)+f(2 x-y)-2 f(x+y)-2 f(x-y)-2[f(2 x)-2 f(x)]
$$

for all $x, y \in X$.

\section{Main results}

In this section, using the direct method, we prove the Hyers-Ulam stability of the functional equation $D_{f}(x, y)=0$ in paranormed spaces. It is easy to check that the mapping $x \mapsto f(x)-f(0)$ also satisfies the equation $D_{f}(x, y)=0$. Thus, throughout this section, without loss of generality, we assume that $f(0)=0$, otherwise we work on the function $x \mapsto f(x)-f(0)$ instead of $f$.

Before proceeding to the proof of the main results, we present the following two lemmas. 
Lemma 3.1 ([9]). Let $\mathrm{V}$ and $\mathrm{W}$ be real vector spaces. If a mapping $\mathrm{f}: \mathrm{V} \rightarrow \mathrm{W}$ satisfies (1.1), then the mapping $\mathrm{g}: \mathrm{V} \rightarrow \mathrm{W}$ defined by $\mathrm{g}(\mathrm{x})=\mathrm{f}(2 \mathrm{x})-2 \mathrm{f}(\mathrm{x})$ is cubic.

Lemma 3.2 ([9]). Let $\mathrm{V}$ and $\mathrm{W}$ be real vector spaces. If a mapping $\mathrm{f}: \mathrm{V} \rightarrow \mathrm{W}$ satisfies (1.1), then the mapping $\mathrm{h}: \mathrm{V} \rightarrow \mathrm{W}$ defined by $\mathrm{h}(\mathrm{x})=\mathrm{f}(2 \mathrm{x})-8 \mathrm{f}(\mathrm{x})$ is additive.

Theorem 3.3. Let $\mathrm{r}$ be positive real number with $\mathrm{r}<3$. Suppose that $\mathrm{f}: \mathrm{X} \rightarrow \mathrm{Y}$ is a mapping satisfying

$$
\left\|D_{f}(x, y)\right\| \leqslant P(x)^{r}+P(y)^{r}
$$

for all $\mathrm{x}, \mathrm{y} \in \mathrm{X}$. Then there exists a unique cubic mapping $\mathrm{C}: \mathrm{X} \rightarrow \mathrm{Y}$ satisfying

$$
\|f(2 x)-2 f(x)-C(x)\| \leqslant \frac{7+2^{r}}{8-2^{r}} P(x)^{r}
$$

for all $x \in X$.

Proof. Setting $x=0$ in (3.1), we have

$$
\|f(y)+f(-y)\| \leqslant P(y)^{r}
$$

for all $y \in X$. Replacing $y$ by $x$ first and then $y$ by $2 x$ in (3.1), we obtain the inequalities

$$
\begin{gathered}
\|f(3 x)-4 f(2 x)+5 f(x)\| \leqslant 2 P(x)^{r} \\
\|f(4 x)-2 f(3 x)-2 f(2 x)-2 f(-x)+4 f(x)\| \leqslant\left(2^{r}+1\right) P(x)^{r}
\end{gathered}
$$

for all $x \in X$. It follows from (3.3), (3.4), and (3.5) that

$$
\begin{aligned}
\|f(4 x)-10 f(2 x)+16 f(x)\| \leqslant & \|2(f(x)+f(-x))\|+\|2(f(3 x)-4 f(2 x)+5 f(x))\| \\
& +\|f(4 x)-2 f(3 x)-2 f(2 x)-2 f(-x)+4 f(x)\| \\
\leqslant & 2 P(x)^{r}+2 \cdot 2 P(x)^{r}+\left(2^{r}+1\right) P(x)^{r}=\left(7+2^{r}\right) P(x)^{r}
\end{aligned}
$$

for all $x \in X$. Defining $g: X \rightarrow Y$ by $g(x):=f(2 x)-2 f(x)$ and using (3.6) we have

$$
\left\|g(x)-\frac{1}{8} g(2 x)\right\| \leqslant \frac{7+2^{r}}{8} P(x)^{r}
$$

for all $x \in X$. Hence

$$
\left\|\frac{1}{8^{m}} g\left(2^{m} x\right)-\frac{1}{8^{n}} g\left(2^{n} x\right)\right\| \leqslant \sum_{j=m}^{n-1} \frac{\left(7+2^{r}\right) 2^{r j}}{8^{j+1}} P(x)^{r}
$$

for all nonnegative integers $n$ and $m$ with $n \geqslant m$ and all $x \in X$. Therefore we conclude from (3.8) that the sequence $\left\{\frac{1}{8^{n}} g\left(2^{n} x\right)\right\}$ is a Cauchy sequence in $Y$ for all $x \in X$. Since $Y$ is complete, the sequence $\left\{\frac{1}{8^{n}} g\left(2^{n} x\right)\right\}$ converges for all $x \in X$. So one can define the mapping $C: X \rightarrow Y$ by

$$
C(x):=\lim _{n \rightarrow \infty} \frac{1}{8^{n}} g\left(2^{n} x\right)
$$

for all $x \in X$. Letting $m=0$ and passing the limit $n \rightarrow \infty$ in (3.8), we get (3.2). Now, we show that the mapping $C$ is cubic. By (3.7) and (3.9),

$$
\begin{aligned}
\|C(2 x)-8 C(x)\| & =\lim _{n \rightarrow \infty}\left\|\frac{1}{8^{n}} g\left(2^{n+1} x\right)-\frac{1}{8^{n-1}} g\left(2^{n} x\right)\right\| \\
& =\lim _{n \rightarrow \infty} \frac{1}{8^{n-1}}\left\|\frac{1}{8} g\left(2^{n+1} x\right)-g\left(2^{n} x\right)\right\| \\
& \leqslant \lim _{n \rightarrow \infty} \frac{\left(7+2^{r}\right) 2^{n r}}{8^{n}} P(x)^{r}=0
\end{aligned}
$$


for all $x \in X$. Therefore, we obtain

$$
\mathrm{C}(2 \mathrm{x})=8 \mathrm{C}(\mathrm{x})
$$

for all $x \in X$. On the other hand it follows from (3.1) and (3.9) that

$$
\begin{aligned}
\left\|D_{C}(x, y)\right\| & =\lim _{n \rightarrow \infty}\left\|\frac{1}{8^{n}} D_{g}\left(2^{n} x, 2^{n} y\right)\right\| \\
& =\lim _{n \rightarrow \infty} \frac{1}{8^{n}}\left\|D_{f}\left(2^{n+1} x, 2^{n+1} y\right)-2 D_{f}\left(2^{n} x, 2^{n} y\right)\right\| \\
& \leqslant \lim _{n \rightarrow \infty} \frac{1}{8^{n}}\left\{2^{n r+r}\left[P(x)^{r}+P(y)^{r}\right]+2^{n r+1}\left[P(x)^{r}+P(y)^{r}\right]\right\} \\
& =\lim _{n \rightarrow \infty} \frac{2^{n r}}{8^{n}}\left(2^{r}+2\right)\left[P(x)^{r}+P(y)^{r}\right]=0
\end{aligned}
$$

for all $x, y \in X$. Hence the mapping $C$ satisfies (1.1). So by Lemma 3.1, the mapping $x \mapsto C(2 x)-2 C(x)$ is cubic. Hence (3.10) implies that the mapping $C$ is cubic. To prove the uniqueness of $C$, let $C^{\prime}: X \rightarrow Y$ be another cubic mapping satisfying (3.2). Then we have

$$
\begin{aligned}
\left\|C(x)-C^{\prime}(x)\right\| & =\left\|\frac{1}{8^{n}} C\left(2^{n} x\right)-\frac{1}{8^{n}} C^{\prime}\left(2^{n} x\right)\right\| \\
& \leqslant\left\|\frac{1}{8^{n}}\left(C\left(2^{n} x\right)-g\left(2^{n} x\right)\right)\right\|+\left\|\frac{1}{8^{n}}\left(C^{\prime}\left(2^{n} x\right)-g\left(2^{n} x\right)\right)\right\| \\
& \leqslant \frac{2\left(7+2^{r}\right) 2^{n r}}{\left(8-2^{r}\right) 8^{n}} P(x)^{r},
\end{aligned}
$$

which tends to zero as $n \rightarrow \infty$ for all $x \in X$. So we can conclude that $C(x)=C^{\prime}(x)$ for all $x \in X$. This completes the proof of the theorem.

Corollary 3.4. Let $\mathrm{r}, \mathrm{s}$ be positive real numbers such that $\rho:=\mathrm{r}+\mathrm{s}<3$. Suppose that $\mathrm{f}: \mathrm{X} \rightarrow \mathrm{Y}$ is a mapping satisfying

$$
\left\|D_{f}(x, y)\right\| \leqslant\left\{\begin{array}{l}
P(x)^{r} P(y)^{s}, \\
P(x)^{r} P(y)^{s}+P(x)^{r+s}+P(y)^{r+s}
\end{array}\right.
$$

for all $\mathrm{x}, \mathrm{y} \in \mathrm{X}$. Then there exists a unique cubic mapping $\mathrm{C}: \mathrm{X} \rightarrow \mathrm{Y}$ satisfying

$$
\|f(2 x)-2 f(x)-C(x)\| \leqslant\left\{\begin{array}{l}
\frac{2+2^{s}}{8-2^{\rho}} P(x)^{\rho} \\
\frac{\left(9+2^{s}\right)+2^{\rho}}{8-2^{\rho}} P(x)^{\rho}
\end{array}\right.
$$

for all $x \in X$.

Proof. The proof is similar to the proof of Theorem 3.3 and thus it is omitted.

Theorem 3.5. Let $r, \theta$ be positive real numbers such that $r>3$. Suppose that $f: Y \rightarrow X$ is a mapping satisfying

$$
P\left(D_{f}(x, y)\right) \leqslant \theta\left(\|x\|^{r}+\|y\|^{r}\right)
$$

for all $\mathrm{x}, \mathrm{y} \in \mathrm{Y}$. Then there exists a unique cubic mapping $\mathrm{C}: \mathrm{Y} \rightarrow \mathrm{X}$ satisfying

$$
P(f(2 x)-2 f(x)-C(x)) \leqslant \frac{2^{r}+7}{2^{r}-8} \theta\|x\|^{r}
$$

for all $\mathrm{x} \in \mathrm{Y}$. 
Proof. Setting $x=0$ in (3.12), we obtain

$$
P(f(y)+f(-y)) \leqslant \theta\|y\|^{r}
$$

for all $y \in Y$. Replacing first $y$ by $x$ and then $y$ by $2 x$ in (3.12), we get

$$
\begin{gathered}
P(f(3 x)-4 f(2 x)+5 f(x)) \leqslant 2 \theta\|x\|^{r}, \\
P(f(4 x)-2 f(3 x)-2 f(2 x)-2 f(-x)+4 f(x)) \leqslant\left(2^{r}+1\right) \theta\|x\|^{r}
\end{gathered}
$$

for all $x \in Y$. It follows from (3.14), (3.15), and (3.16) that

$$
\begin{aligned}
P(f(4 x)-10 f(2 x)+16 f(x)) \leqslant & P(2(f(x)+f(-x)))+P(2(f(3 x)-4 f(2 x)+5 f(x))) \\
& +P(f(4 x)-2 f(3 x)-2 f(2 x)-2 f(-x)+4 f(x)) \\
\leqslant & 2 \theta\|x\|^{r}+2 \cdot 2 \theta\|x\|^{r}+\left(2^{r}+1\right) \theta\|x\|^{r} \\
= & \left(2^{r}+7\right) \theta\|x\|^{r}
\end{aligned}
$$

for all $x \in Y$. Defining $g: Y \rightarrow X$ by $g(x):=f(2 x)-2 f(x)$ and using (3.17) we have

$$
P\left(g(x)-8 g\left(\frac{x}{2}\right)\right) \leqslant \frac{2^{r}+7}{2^{r}} \theta\|x\|^{r}
$$

for all $x \in Y$. Hence

$$
P\left(8^{m} g\left(\frac{x}{2^{m}}\right)-8^{n} g\left(\frac{x}{2^{n}}\right)\right) \leqslant \sum_{j=m}^{n-1} \frac{\left(2^{r}+7\right) 8^{j}}{2^{r j+r}} \theta\|x\|^{r}
$$

for all nonnegative integers $n$ and $m$ with $n \geqslant m$ and all $x \in Y$. It follows from (3.19) that the sequence $\left\{8^{n} g\left(\frac{x}{2^{n}}\right)\right\}$ is a Cauchy sequence in $X$ for all $x \in Y$. Since $X$ is complete, the sequence $\left\{8^{n} g\left(\frac{x}{2^{n}}\right)\right\}$ converges for all $x \in Y$. So one can define the mapping $C: Y \rightarrow X$ by

$$
C(x):=\lim _{n \rightarrow \infty} 8^{n} g\left(\frac{x}{2^{n}}\right)
$$

for all $x \in Y$. Moreover, letting $m=0$ and passing the limit $n \rightarrow \infty$ in (3.19), we get (3.13). Now, we show that the mapping $C$ is cubic. By (3.18) and (3.20),

$$
\begin{aligned}
P\left(C(x)-8 C\left(\frac{x}{2}\right)\right) & =\lim _{n \rightarrow \infty} P\left(8^{n} g\left(\frac{x}{2^{n}}\right)-8^{n+1} g\left(\frac{x}{2^{n+1}}\right)\right) \\
& =\lim _{n \rightarrow \infty} 8^{n} P\left(g\left(\frac{x}{2^{n}}\right)-8 g\left(\frac{x}{2^{n+1}}\right)\right) \\
& \leqslant \lim _{n \rightarrow \infty} \frac{\left(2^{r}+7\right) 8^{n}}{2^{n+r}} \theta\|x\|^{r}=0
\end{aligned}
$$

for all $x \in Y$. Therefore, we obtain

$$
C(x)=8 C\left(\frac{x}{2}\right)
$$

for all $x \in Y$. On the other hand it follows from (3.12) and (3.20) that

$$
\begin{aligned}
P\left(D_{C}(x, y)\right) & =\lim _{n \rightarrow \infty} P\left(8^{n} D_{g}\left(\frac{x}{2^{n}}, \frac{y}{2^{n}}\right)\right) \\
& =\lim _{n \rightarrow \infty} 8^{n} P\left(D_{f}\left(\frac{x}{2^{n-1}}, \frac{y}{2^{n-1}}\right)-2 D_{f}\left(\frac{x}{2^{n}}, \frac{y}{2^{n}}\right)\right) \\
& \leqslant \lim _{n \rightarrow \infty} \frac{8^{n}}{2^{n r}}\left(2^{r}+2\right) \theta\left(\|x\|^{r}+\|y\|^{r}\right)=0
\end{aligned}
$$


for all $x, y \in Y$. Hence the mapping $C$ satisfies (1.1). So by Lemma 3.1, the mapping $x \mapsto C(2 x)-2 C(x)$ is cubic. Hence (3.21) implies that the mapping $C$ is cubic. To prove the uniqueness of $C$, let $C^{\prime \prime}: Y \rightarrow X$ be another cubic mapping satisfying (3.13). Then we have

$$
\begin{aligned}
P\left(C(x)-C^{\prime \prime}(x)\right) & =P\left(8^{n} C\left(\frac{x}{2^{n}}\right)-8^{n} C^{\prime \prime}\left(\frac{x}{2^{n}}\right)\right) \\
& \leqslant 8^{n}\left(P\left(C\left(\frac{x}{2^{n}}\right)-g\left(\frac{x}{2^{n}}\right)\right)+P\left(C^{\prime \prime}\left(\frac{x}{2^{n}}\right)-g\left(\frac{x}{2^{n}}\right)\right)\right) \\
& \leqslant \frac{2\left(2^{r}+7\right) 8^{n}}{\left(2^{r}-8\right) 2^{n r}} \theta\|x\|^{r},
\end{aligned}
$$

which tends to zero as $n \rightarrow \infty$ for all $x \in Y$. So we can conclude that $C(x)=C^{\prime \prime}(x)$ for all $x \in Y$. This completes the proof of the theorem.

Corollary 3.6. Let $r, s, \theta$ be positive real numbers such that $\rho:=r+s>3$. Suppose that $f: Y \rightarrow X$ is a mapping satisfying

$$
P\left(D_{f}(x, y)\right) \leqslant\left\{\begin{array}{l}
\theta\|x\|^{r}\|y\|^{s}, \\
\theta\left(\|x\|^{r}\|y\|^{s}+\left(\|x\|^{r+s}+\|y\|^{r+s}\right)\right)
\end{array}\right.
$$

for all $\mathrm{x}, \mathrm{y} \in \mathrm{Y}$. Then there exists a unique cubic mapping $\mathrm{C}: \mathrm{Y} \rightarrow \mathrm{X}$ satisfying

$$
P(f(2 x)-2 f(x)-C(x)) \leqslant\left\{\begin{array}{l}
\frac{2^{s}+2}{2^{\rho}-8} \theta\|x\|^{\rho} \\
\frac{2^{\rho}+\left(2^{s}+9\right)}{2^{\rho}-8} \theta\|x\|^{\rho}
\end{array}\right.
$$

for all $\mathrm{x} \in \mathrm{Y}$.

Proof. The proof is similar to the proof of Theorem 3.5 and thus it is omitted.

Theorem 3.7. Let $\mathrm{r}$ be positive real number with $\mathrm{r}<1$. Suppose that $\mathrm{f}: \mathrm{X} \rightarrow \mathrm{Y}$ is a mapping satisfying

$$
\left\|D_{f}(x, y)\right\| \leqslant P(x)^{r}+P(y)^{r}
$$

for all $x, y \in X$. Then there exists a unique additive mapping $A: X \rightarrow Y$ satisfying

$$
\|f(2 x)-8 f(x)-A(x)\| \leqslant \frac{7+2^{r}}{2-2^{r}} P(x)^{r}
$$

for all $x \in X$.

Proof. The proof is similar to the proof of Theorem 3.3 and thus it is omitted.

Corollary 3.8. Let $r, s$ be positive real numbers such that $\rho:=r+s<1$. Suppose that $f: X \rightarrow Y$ is a mapping satisfying (3.11) for all $x, y \in X$. Then there exists a unique additive mapping $A: X \rightarrow Y$ satisfying

$$
\|f(2 x)-8 f(x)-A(x)\| \leqslant\left\{\begin{array}{l}
\frac{2+2^{s}}{2-2^{\rho}} P(x)^{\rho} \\
\frac{\left(2^{s}+9\right)+2^{\rho}}{2-2^{\rho}} P(x)^{\rho}
\end{array}\right.
$$

for all $x \in X$.

Proof. The proof is similar to the proof of Theorem 3.7 and thus it is omitted.

Theorem 3.9. Let $r, \theta$ be positive real numbers such that $r>1$. Suppose that $f: Y \rightarrow X$ is a mapping satisfying

$$
P\left(D_{f}(x, y)\right) \leqslant \theta\left(\|x\|^{r}+\|y\|^{r}\right)
$$

for all $x, y \in Y$. Then there exists a unique additive mapping $A: Y \rightarrow X$ satisfying

for all $\mathrm{x} \in \mathrm{Y}$.

$$
P(f(2 x)-8 f(x)-A(x)) \leqslant \frac{2^{r}+7}{2^{r}-2} \theta\|x\|^{r}
$$


Proof. The proof is similar to the proof of Theorem 3.5 and thus it is omitted.

Corollary 3.10. Let $\mathrm{r}, \mathrm{s}, \theta$ be positive real numbers such that $\rho:=\mathrm{r}+\mathrm{s}>1$. Suppose that $\mathrm{f}: \mathrm{Y} \rightarrow \mathrm{X}$ is a mapping satisfying (3.22) for all $x, y \in Y$. Then there exists a unique additive mapping $A: Y \rightarrow X$ satisfying

$$
P(f(2 x)-8 f(x)-A(x)) \leqslant\left\{\begin{array}{l}
\frac{2^{s}+2}{2^{\rho}-2} \theta\|x\|^{\rho} \\
\frac{2^{\rho}+\left(2^{s}+9\right)}{2^{\rho}-2} \theta\|x\|^{\rho}
\end{array}\right.
$$

for all $\mathrm{x} \in \mathrm{Y}$.

Proof. The proof is similar to the proof of Theorem 3.9 and thus it is omitted.

Applying Theorems 3.3 and 3.7, we obtain the following result.

Theorem 3.11. Let $\mathrm{r}$ be positive real number with $\mathrm{r}<1$. Suppose that $\mathrm{f}: \mathrm{X} \rightarrow \mathrm{Y}$ is a mapping satisfying (3.1). Then there exist a unique additive mapping $\mathrm{A}: \mathrm{X} \rightarrow \mathrm{Y}$ and a unique cubic mapping $\mathrm{C}: \mathrm{X} \rightarrow \mathrm{Y}$ such that

$$
\|6 f(x)-A(x)-C(x)\| \leqslant\left(\frac{7+2^{r}}{2-2^{r}}+\frac{7+2^{r}}{8-2^{r}}\right) P(x)^{r}
$$

for all $x \in X$.

Proof. Let $g(x)=f(2 x)-2 f(x)$ and $h(x)=f(2 x)-8 f(x)$. Then $6 f(x)=g(x)-h(x)$. By Theorems 3.3 and 3.7, there exists an additive mapping $A_{0}: X \rightarrow Y$ and a cubic mapping $C_{0}: X \rightarrow Y$ such that

$$
\left\|f(2 x)-8 f(x)-A_{0}(x)\right\| \leqslant \frac{7+2^{r}}{2-2^{r}} P(x)^{r}, \quad\left\|f(2 x)-2 f(x)-C_{0}(x)\right\| \leqslant \frac{7+2^{r}}{8-2^{r}} P(x)^{r}
$$

for all $x \in X$. Therefore it follows from the last inequalities that

$$
\begin{aligned}
\left\|6 f(x)+A_{0}(x)-C_{0}(x)\right\| & =\left\|g(x)-h(x)+A_{0}(x)-C_{0}(x)\right\| \\
& \leqslant\left\|h(x)-A_{0}(x)\right\|+\left\|g(x)-C_{0}(x)\right\| \\
& \leqslant\left(\frac{7+2^{r}}{2-2^{r}}+\frac{7+2^{r}}{8-2^{r}}\right) P(x)^{r}
\end{aligned}
$$

for all $x \in X$. So we obtain (3.23) by letting $A(x)=-A_{0}(x)$ and $C(x)=C_{0}(x)$ for all $x \in X$.

To prove the uniqueness of $A$ and $C$, let $A_{1}, C_{1}: X \rightarrow Y$ be another additive and cubic mappings satisfying (3.23), and let $A^{\prime}=A-A_{1}$ and $C^{\prime}=C-C_{1}$. So

$$
\left\|A^{\prime}(x)+C^{\prime}(x)\right\| \leqslant\|6 f(x)-A(x)-C(x)\|+\left\|6 f(x)-A_{1}(x)-C_{1}(x)\right\| \leqslant 2\left(\frac{7+2^{r}}{2-2^{r}}+\frac{7+2^{r}}{8-2^{r}}\right) P(x)^{r}
$$

for all $x \in X$. Since

$$
\lim _{n \rightarrow \infty} \frac{1}{8^{n}} P\left(2^{n} x\right)^{r}=\lim _{n \rightarrow \infty} \frac{1}{2^{n}} P\left(2^{n} x\right)^{r}=0
$$

for all $x \in X,(3.24)$ implies

$$
\lim _{n \rightarrow \infty} \frac{1}{8^{n}}\left\|A^{\prime}\left(2^{n} x\right)+C^{\prime}\left(2^{n} x\right)\right\|=0
$$

for all $x \in X$. Therefore $C^{\prime}(x)=0$ for all $x \in X$. By (3.24), we have $A^{\prime}(x)=0$ for all $x \in X$. This completes the proof of the theorem. 
Corollary 3.12. Let $\mathrm{r}, \mathrm{s}$ be positive real numbers such that $\rho:=\mathrm{r}+\mathrm{s}<1$. Suppose that $\mathrm{f}: \mathrm{X} \rightarrow \mathrm{Y}$ is a mapping satisfying (3.11) for all $\mathrm{x}, \mathrm{y} \in \mathrm{X}$. Then there exist a unique additive mapping $\mathrm{A}: \mathrm{X} \rightarrow \mathrm{Y}$ and a unique cubic mapping $\mathrm{C}: \mathrm{X} \rightarrow \mathrm{Y}$ such that

$$
\|6 f(x)-A(x)-C(x)\| \leqslant\left\{\begin{array}{l}
\left(\frac{2+2^{s}}{8-2^{\rho}}+\frac{2+2^{s}}{2-2^{\rho}}\right) P(x)^{\rho} \\
\left(\frac{\left(9+2^{s}\right)+2^{\rho}}{8-2^{\rho}}+\frac{\left(9+2^{s}\right)+2^{\rho}}{2-2^{\rho}}\right) P(x)^{\rho}
\end{array}\right.
$$

for all $x \in X$.

Proof. The result follows from Theorem 3.11.

Similar to Theorems 3.5 and 3.9, we also obtain the following results.

Theorem 3.13. Let $r, \theta$ be positive real numbers such that $r>3$. Suppose that $f: Y \rightarrow X$ is a mapping satisfying (3.12). Then there exist a unique additive mapping $\mathrm{A}: \mathrm{Y} \rightarrow \mathrm{X}$ and a unique cubic mapping $\mathrm{C}: \mathrm{X} \rightarrow \mathrm{Y}$ such that

$$
P(6 f(x)-A(x)-C(x)) \leqslant\left(\frac{2^{r}+7}{2^{r}-2}+\frac{2^{r}+7}{2^{r}-8}\right) \theta\|x\|^{r}
$$

for all $\mathrm{x} \in \mathrm{Y}$.

Proof. The proof is similar to the proof of Theorem 3.11 and the result follows from Theorems 3.5 and 3.9.

Corollary 3.14. Let $\mathrm{r}, \mathrm{s}, \theta$ be positive real numbers such that $\rho:=\mathrm{r}+\mathrm{s}>3$. Suppose that $\mathrm{f}: \mathrm{Y} \rightarrow \mathrm{X}$ is a mapping satisfying (3.22) for all $\mathrm{x}, \mathrm{y} \in \mathrm{Y}$. Then there exist a unique additive mapping $\mathrm{A}: \mathrm{X} \rightarrow \mathrm{Y}$ and a unique cubic mapping $\mathrm{C}: \mathrm{X} \rightarrow \mathrm{Y}$ such that

$$
P(6 f(x)-A(x)-C(x)) \leqslant\left\{\begin{array}{l}
\left(\frac{2^{s}+2}{2^{\rho}-8}+\frac{2^{s}+2}{2^{\rho}-2}\right) \theta\|x\|^{\rho} \\
\left(\frac{2^{\rho}+\left(2^{s}+9\right)}{2^{\rho}-8}+\frac{2^{\rho}+\left(2^{s}+9\right)}{2^{\rho}-2}\right) \theta\|x\|^{\rho}
\end{array}\right.
$$

for all $\mathrm{x} \in \mathrm{Y}$.

Proof. The result follows from Theorem 3.13.

\section{Acknowledgment}

The authors are thankful to referees for their valuable suggestions that improved the presentation of the paper. This research was done during the first author's visit to the University of Louisville as a Visiting Scholar in 2015-16. The first author is supported by National Natural Science Foundation of China (Grant Nos. 11401190).

\section{References}

[1] T. Aoki, On the stability of the linear transformation in Banach spaces, J. Math. Soc. Japan, 2 (1950), 64-66. 1

[2] G. L. Forti, The stability of homomorphisms and amenability, with applications to functional equations, Abh. Math. Sem. Univ. Hamburg, 57 (1987), 215-226. 1

[3] P. Găvruţa, A generalization of the Hyers-Ulam-Rassias stability of approximately additive mappings, J. Math. Anal. Appl., 184 (1994), 431-436. 1

[4] D. H. Hyers, On the stability of the linear functional equation, Proc. Nat. Acad. Sci. U. S. A., 27 (1941), $222-224.1$

[5] D. H. Hyers, G. Isac, T. M. Rassias, Stability of functional equations in several variables, Progress in Nonlinear Differential Equations and their Applications, Birkhäuser Boston, Inc., Boston, MA, (1998). 1

[6] S.-M. Jung, Hyers-Ulam-Rassias stability of functional equations in nonlinear analysis, Springer Optimization and Its Applications, Springer, New York, (2011). 
[7] P. Kannappan, Functional equations and inequalities with applications, Springer Monographs in Mathematics, Springer, New York, (2009). 1

[8] S. Lee, C. Park, J. R. Lee, Functional inequalities in paranormed spaces, J. Chungcheong Math. Soc., 26 (2013), $287-296$. 1

[9] A. Najati, G. Z. Eskandani, Stability of a mixed additive and cubic functional equation in quasi-Banach spaces, J. Math. Anal. Appl., 342 (2008), 1318-1331. 1, 3.1, 3.2

[10] C. Park, Stability of an AQCQ-functional equation in paranormed spaces, Adv. Difference Equ., 2012 (2012), 20 pages. 1

[11] C. Park, J. R. Lee, An AQCQ-functional equation in paranormed spaces, Adv. Difference Equ., 2012 (2012), 9 pages.

[12] C. Park, J. R. Lee, Functional equations and inequalities in paranormed spaces, J. Inequal. Appl., 2013 (2013), 23 pages.

[13] C. Park, D. Y. Shin, Functional equations in paranormed spaces, Adv. Difference Equ., 2012 (2012), 14 pages. 1

[14] T. M. Rassias, On the stability of the linear mapping in Banach spaces, Proc. Amer. Math. Soc., 72 (1978), 297-300. 1

[15] J. M. Rassias, On approximation of approximately linear mappings by linear mappings, J. Funct. Anal., 46 (1982), $126-130$. 1

[16] T. M. Rassias (Ed.), Functional equations, inequalities and applications, Kluwer Academic Publishers, Dordrecht, (2003). 1

[17] P. K. Sahoo, P. Kannappan, Introduction to functional equations, CRC Press, Boca Raton, FL, (2011). 1

[18] S. M. Ulam, Problems in modern mathematics, Science Editions John Wiley \& Sons, Inc., New York, (1964). 1 